\title{
Actitudes y Uso del Lenguaje Inclusivo segun el Género y la Edad
}

\section{Attitudes and Use of Inclusive Languageaccording to Gender and Age}

\author{
Agustina Pesce \\ $\triangle$ agustinapesce@economicas.uba.ar \\ iD http://orcid.org/0000-0003-2907-8429

\section{Edgardo Etchezahar} \\ Universidad de Buenos Aires. Facultad de Psicología. \\ CIIPME (CONICET). Buenos Aires, Argentina \\ Universidad Nacional de Lomas de Zamora. \\ Facultad de Ciencias Sociales. Buenos Aires, Argentina \\ (iD) http://orcid.org/0000-0002-3289-194X
}

Universidad de Buenos Aires. Facultad de Psicología. Buenos Aires, Argentina

Recibido: 21-09-2019

Aceptado: 07-12-2019

\section{RESUMEN}

El objetivo del presente trabajo fue describir la relación entre las actitudes y uso del Lenguaje Inclusivo (LI) con las variables demográficas género y edad. El Lenguaje Inclusivo es aquel uso del lenguaje que no reconoce al género masculino como genérico de la humanidad, buscando reducir los estereotipos y discriminación a través de la comunicación. Para ello, se trabajó con un diseño cuantitativo, ex post facto prospectivo de grupo único, en el se analizaron las respuestas a un cuestionario autoadministrable de 613 participantes, residentes en la Ciudad Autónoma de Buenos Aires, obtenidas a través de un muestreo no probabilístico e intencional. El rango de edades fue de 18 a 70 años; y la distribución de los géneros de 346 participantes femeninas, 256 masculinos y 11 no binariosm, a través de un método ex post facto prospectivo. En los resultados se hallaron diferencias, según el Género, para las actitudes positivas hacia el Lenguaje Inclusivo y para la frecuencia de su uso. Las participantes de género femenino tendrían una mayor valoración positiva del Lenguaje Inclusivo, y lo utilizarían más respecto a aquellos de género masculino. Por otro lado, en la franja etaria más baja (18 a 23 años) y más alta (50 a 70 años), se observaron menores actitudes positivas hacia el Lenguaje Inclusivo, mientras que los grupos de edades intermedias obtuvieron valores más elevados para esta variable. Es necesario continuar estudiando los alcances y límites del Lenguaje Inclusivo en otros factores psicosociales.

Palabras clave: Género; lenguaje inclusivo; edad; actitudes. 


\section{ABSTRACT}

The objective of this paper was to describe the relationship between attitudes and use of Inclusive Language (LI) with the demographic variables Gender and Age. Inclusive Language is that use of language that does not recognize the male gender as a generic of humanity, seeking to reduce stereotypes and discrimination through communication. For this, we work whit a cuantitative research design ex post facto and prospective research whit one group design and the responses to a self-administered questionnaire of 613 participants, residents of the city of Buenos Aires, obtained through a nonprobabilistic and intentional sampling were analyzed. The age range was 18 to 70 years; and gender distribution of 346 female, 256 male and 11 non-binary participants, through a prospective ex post facto method. In the results, differences were found, according to gender, for positive attitudes towards Inclusive Language and for the frequency of its use. Female participants would have a higher positive assessment of Inclusive Language, and would use it more than those of male gender. On the other hand, in the lowest age range (18 to 23 years old) and highest ( 50 to 70 years old), lower positive attitudes towards Inclusive Language were observed, while the intermediate age groups obtained higher values for this variable. It is necessary to continue studying the scope and limits of Inclusive Language in other psychosocial factors.

Keywords: Gender; inclusive language; age; attitudes.

\section{INTRODUCCIÓN}

El Lenguaje Inclusivo es aquel uso del lenguaje en el cual no se reconoce al Género masculino como genérico de la humanidad (Chenlo, 2014). Su utilización se orienta a la superación de la asimetría estructural de género que presentan muchos idiomas, intentando confrontar los estereotipos y la discriminación que se transmite a través del lenguaje (Sczesny, Formanowicz y Moser, 2016).

Las lenguas con género gramatical -como el español- utilizan atribuciones de Género arbitrarias en un amplio espectro de sus mensajes. Sin embargo, el Lenguaje Inclusivo es utilizado solo cuando se refiere a personas, no así cuando se habla de animales o cosas (Chenlo, 2014). Algunas de las novedades introducidas por este uso del lenguaje es mencionar la doble forma femenino y masculino (también llamada feminización), utilizar términos más neutros como "personas" o "ciudadanía" (neutralización), o colocar un "@", asterisco, "x" o "e" en la vocal que indica el Género gramatical de las palabras.

Otro término en español para referirse a este concepto es lenguaje no sexista (Bejarano, 2013; Jiménez Rodrigo, Onsalo y Traverso Cortés, 2011), el cual apunta, igualmente, a no reconocer al Género masculino como genérico. Por otro lado, en inglés, principalmente se emplea el término lenguaje justo de Género (gender-fair language) (Formanowicz, Cislak, Horvath y Sczesny, 2015; Gustafsson Sendén, Bäck y Lindqvist, 2015; Lindqvist, Renström y Sendén, 2018; Sczesny et al., 2016).

En este trabajo se utilizará la denominación Lenguaje Inclusivo, por representar la forma más difundida con que se lo conoce en Argentina, pero, también, porque evita las posibles connotaciones biologicistas del término "sexista" y su correspondiente binarismo.

Los efectos psicológicos, implicados en la inclusión del género gramatical en el lenguaje, tienen una larga trayectoria en la investigación empírica (Sato y Athanasopoulos, 2018). Específicamente, en lo que refiere al Lenguaje Inclusivo, existe una serie de estudios 
que indican que este produce cambios en las representaciones de quienes reciben el mensaje, en comparación con sus alternativas en genérico masculino. Por ejemplo, en un estudio se preguntó a diferentes personas respecto a sus personalidades preferidas de ficción (e.g. héroe o heroína de novela) y de la realidad (e.g. pintor o pintora) con tres versiones: utilizando el masculino como genérico, fórmulas neutrales, o tanto el sustantivo femenino como el masculino. Como resultado, se observó un aumento significativo de las mujeres mencionadas en las condiciones de neutralización y feminización, por sobre aquellas donde se implementaba el masculino como genérico (Stahlberg y Sczesny, 2001). A partir de estos resultados, podría pensarse que la utilización de este último volvería más costosa la evocación de personas y personajes femeninos.

Otros trabajos indican que el uso del masculino como genérico tiene consecuencias prácticas en el ámbito laboral. Stout y Dasgupta (2011) Ilevaron a cabo tres estudios, donde se describía un trabajo a participantes mujeres y hombres utilizando el masculino como genérico, términos neutrales o términos en ambos géneros. Tras analizar la percepción de sí en función del trabajo, se halló que las mujeres tenían un menor sentimiento de pertenencia, motivación e identificación con el mismo cuando no se incluían la forma neutral o feminizada en el discurso. También, dentro del ámbito laboral, una investigación realizada con estudiantes de escuela primaria indicaría que, cuando a esta población se le presentan nombres de ocupaciones en Género Femenino y masculino, la accesibilidad de personas femeninas ejerciendo esos trabajos es más balanceada respecto a cuando solo se menciona en genérico masculino (Vervecken, Hannover y Wolter, 2013).

Si bien, la mayor parte de las investigaciones empíricas acerca de la utilización del masculino como genérico (y sus alternativas inclusivas), fueron realizadas en hablantes del idioma inglés y alemán, es esperable que el patrón de resultados sea el mismo para hispanohablantes, ya que tienen formas similares para enunciar los genéricos y resolver los sesgos lingüísticos (Kaufmann y Bohner, 2014).

Las investigaciones mencionadas revelan algunas de las formas en las cuales el lenguaje modela el pensamiento, haciendo un aporte en la construcción de la realidad de quienes participaron en las mismas (Jiménez Rodrigo et al., 2010, 2011). Al aprender un lenguaje se incorporan, de manera imperceptible, ciertos sentidos y estereotipos provenientes del contexto en el cual fue acuñado. Esto lleva a que un lenguaje como el español, con origen en contextos donde la repartición de tareas en la esfera pública y privada estaba y continúa estando ligada al género (Aguirre y Ferrari, 2014), contenga sesgos producto de este régimen de discursos y prácticas, reflejando una mayor posición de poder para los individuos masculinos (Campagnoli, 2014).

Las implicaciones psicológicas que tiene el uso del masculino como genérico, el cual lleva a la sobrerrepresentación del Género masculino sobre los otros géneros, sería efecto de la forma difusa y ubicua en la cual el lenguaje transmite sus estereotipos, careciendo de responsables directos e involucrando a toda la trama social (Campagnoli, 2014). Para reducir y hasta eliminar este sesgo, es necesaria una compleja modificación de nuestra sociedad, que cambie las estructuras sociales discriminatorias (Campagnoli, 2014; Jiménez Rodrigo et al., 2010, 2011; Puppo, 2009). En este sentido, los cambios en el lenguaje pueden llevar a la visibilización de las disparidades de género, resaltando la inequidad existente en ciertos ámbitos y conduciendo a la búsqueda de su transformación (Kaufmann, y Bohner, 2014).

EI Género y la Edad en las Actitudes hacia el Lenguaje Inclusivo. Estudiar las relaciones entre variables demográficas y el uso del Lenguaje Inclusivo es importante, debido a que el lenguaje, como herramienta dentro de las prácticas sociales, funciona 
como un dispositivo, no solo para transferir información, sino también para expresar categorizaciones sociales y jerarquías, lo que lo vuelve un medio para reducir los sesgos de Género (Maas y Arcuri, 1996; Sczesny, Moser y Wood, 2015).

Otro constructo de relevancia para ser evaluado es el de actitudes positivas hacia el Lenguaje Inclusivo. Las actitudes refieren a disposiciones favorables o desfavorables hacia objetos sociales, como personas, lugares o políticas (Greenwald y Benaji, 1995). Dicha evaluación puede referirse al objeto en sí mismo, o bien, a sus consecuencias. Si bien la conducta es influenciada por una amplia cantidad de factores (como las normas sociales y el nivel de control conductual percibido), las actitudes permiten evaluar cuáles son las valoraciones hacia Lenguaje Inclusivo vigentes en la sociedad, más allá de la difusión de su uso (Knussen, Yule, MacKenzie y Wells, 2004; Fishbein y Ajzen, 2011; Sczesny et al., 2015).

Las investigaciones empíricas sobre Lenguaje Inclusivo -y su uso diferencial en la población - tienen una historia de al menos cuarenta años en los estudios sobre uso de pronombres en lengua inglesa (Martyna, 1978). Luego, estos también empezaron a tener lugar en Europa, en distintas lenguas romances y escandinavas (Gustafsson Sendén et al., 2015; Sarrasin, Gabriel y Gygax, 2012; Sczesny et al., 2015). Las primeras variables que se han tenido en cuenta para analizar la relación con los constructos mencionados en estas investigaciones fueron el género y la edad de sus participantes.

En lo que refiere al género, Parks y Roberton (1998) encontraron, en su estudio de apoyo al Lenguaje Inclusivo, una diferencia de medias entre los grupos de diferente Género ( $d$ de Cohen $=.69$ ), donde el grupo de participantes femeninas apoyó su uso en mayor medida. En el mismo sentido, en otro estudio más actual realizado en los Estados Unidos (con participantes de entre 18 y 30 años), se obtuvieron resultados que indicarían un apoyo al Lenguaje Inclusivo significativamente mayor por parte de las participantes femeninas respecto a los masculinos (Douglas y Sutton, 2014). Por otro lado, en una investigación realizada a lo largo de cuatro años en Suecia (Gustafsson Sendén, et al. 2015), se halló que la relación del Género con las actitudes hacia el Lenguaje Inclusivo perdía fuerza cuando se introducían al modelo de regresión variables como el sexismo y la orientación política. En cuanto al uso del Lenguaje Inclusivo, el Género predijo la conducta en todos los modelos, excepto en aquel donde se evaluaba también el interés en cuestiones de género y en la importancia dada a la propia identidad de género (Gustafsson Sendén et al., 2015). Esto indicaría que, al menos en el contexto donde se desarrolló el estudio, el género de las personas influye en las valoraciones y uso del Lenguaje Inclusivo de forma limitada. Por último, en una investigación realizada en habla alemana, con participantes de entre 18 y 54 años, no se hallaron diferencias entre los géneros femenino y masculino para el uso ni para las actitudes hacia el Lenguaje Inclusivo (Sczesny et al., 2015).

En este trabajo, el género será entendido como aquel sentimiento que se detenta sobre la categoría de Género que auténticamente representa quién se es (Hyde, Bigler, Joel, Tate y van Anders, 2018). Este se encuentra entrelazado con niveles representacionales, interaccionales y con procesos estructurales de la sociedad (Budgeon, 2013). Dada esta multidimensionalidad $-y$ debido al creciente número de investigaciones que desafían el binarismo de Género desde múltiples marcos teóricos (Hyde et al., 2018) - , se decidió tener en cuenta tres categorías: "femenino", "masculino" y "no binario". Si bien no es posible abarcar con un único término la diversidad de las identidades no cisgénero (Frohard-Dourlent, Dobson, Clark, Doull y Saewyc, 2017), para el propósito de esta investigación, se utilizará el término "no binario" para incluir aquellas identidades de género que no se reconocen en las categorías "femenino" o "masculino". 
En cuanto a las relaciones del Lenguaje Inclusivo con la edad, las indagaciones realizadas hasta el momento han tenido resultados disímiles. En el trabajo de Parks y Roberton (1998), el grupo de más de 23 años mostró una mayor aceptación del Lenguaje Inclusivo por sobre el grupo de entre 18 y 23 años. En una investigación, años más tarde, estas mismas investigadoras encontraron una diferencia de opiniones aún mayor entre mujeres y varones, cuando restringieron su población a estudiantes más jóvenes, de entre 18 y 22 años ( $d$ de Cohen $=.88$ ), es decir que a menor edad las opiniones hacia el Lenguaje Inclusivo se mostraron más polarizadas según el Género (Parks y Roberton, 2008). Como posibles fundamentos para estos resultados, conjeturaron que la edad podría llevar a un incremento de la empatía hacia las otras personas, o bien, que la vivencia de determinados hechos históricos por el grupo de mayor edad habría conducido a una visión más igualitaria de la sociedad. Por último, en la investigación realizada en Suecia, mencionada anteriormente, la edad tuvo un poder explicativo para las actitudes y uso del Lenguaje Inclusivo en todos los modelos de regresión, donde a mayor edad se observaron menores actitudes positivas y un menor uso del Lenguaje Inclusivo (Gustafsson Sendén et al., 2015). La circunscripción de las muestras a instituciones universitarias o a grupos de participantes de la misma edad ha hecho que esta variable haya sido analizada en pocas ocasiones en relación al Lenguaje Inclusivo.

Investigaciones, en contextos donde la discusión sobre el Lenguaje Inclusivo tiene una mayor tradición, reflejan la relevancia que tiene el transcurso del tiempo para la aceptación de la introducción de términos inclusivos novedosos. En Suecia, el rechazo inicial al uso de un nuevo pronombre neutro en 2012 cambió rápidamente para 2015, donde las manifestaciones "muy negativas" sobre su introducción fueron bajas (Gustafsson Sendén et al., 2015). Iguales conclusiones fueron obtenidas en investigaciones donde se compararon contextos donde el Lenguaje Inclusivo se encontraba firmemente establecido, respecto a otros, donde su incorporación era reciente o escasa (Sarrasin, Gabriel y Gygax, 2012). Como explica Gustafsson Sendén (2015) resultados como estos representan un incentivo para propiciar cambios en el lenguaje, aun cuando en un principio las reacciones no sean favorables.

A pesar de la incipiente introducción de este uso del lenguaje en Argentina, hasta el momento no se ha analizado si su uso y las actitudes hacia el mismo guardan una relación con el género y la edad de las personas. Esta información, la cual ha tenido diferentes resultados en los estudios realizados hasta el momento, sería de gran ayuda para la ideación de estrategias que concienticen sobre sus ventajas e incentiven su uso. El objetivo de este trabajo fue describir cuál es la relación entre el uso y las actitudes hacia el Lenguaje Inclusivo con la edad y el género de individuos de la Ciudad Autónoma de Buenos Aires. Como hipótesis, se planteó que las participantes femeninas y de Géneros no binarios tendrían una actitud más positiva y un mayor uso del Lenguaje Inclusivo, en comparación con aquellos que se identifican con el género masculino. Asimismo, se hipotetizó que la variable edad se relacionaría de forma inversa con las actitudes positivas y el uso del Lenguaje Inclusivo.

\section{MÉTODO}

Participantes. Se llevó a cabo un muestreo no probabilístico e intencional compuesto por 613 participantes residentes de la Ciudad Autónoma de Buenos Aires. El rango etario fue de 18 a 70 años $(M=32.75 ; D T=12.45)$. La distribución, según el género, fue de 346 participantes femeninas, 256 masculinos y 11 no binarios. 
Instrumentos. Los datos se obtuvieron a partir de un instrumento de tipo autoadministrable el cual incluía las siguientes variables:

Actitudes Positivas hacia el Lenguaje Inclusivo. Se utilizó una escala de ocho ítems extraída del estudio desarrollado por de Sczesny et al. (2015). Los ítems fueron traducidos del inglés de manera directa y su equivalencia fue evaluada por dos hablantes de inglés y español. El grado de acuerdo se evaluó con una escala Likert de siete niveles, siendo el nivel más bajo "Completamente en Desacuerdo" y el más alto "Completamente de Acuerdo". Dos ejemplos de items de esta escala son "El Lenguaje Inclusivo es una cuestión de relevancia personal para mí" y "El Lenguaje Inclusivo es algo que ayuda a la igualdad de género". La consistencia interna de la escala fue adecuada $(a=.926)$.

Uso del Lenguaje Inclusivo. El uso del Lenguaje Inclusivo se evaluó a partir de tres ítems que poseían un formato de respuesta tipo Likert de cinco anclajes siendo el nivel más bajo "Nunca" y el más alto "Muy Frecuentemente". Mientras el primer ítem indagaba sobre el constructo de manera global, el segundo apuntaba al uso escrito y el tercero al uso oral. La consistencia interna de la escala fue adecuada ( $a=.960)$.

Variables Demográficas. La edad fue determinada a través de un espacio en blanco donde se podía ingresar el número correspondiente. Por otro lado, para la identidad de género, el instrumento permitía elegir entre tres opciones posibles: "femenino", "masculino" o "no binario".

Procedimientos. Se invitó a múltiples personas a responder la encuesta de forma voluntaria. La misma era anónima y se indicó a quienes participaron que los resultados iban a ser utilizados con una finalidad académica, dando su consentimiento. La encuesta fue administrada a lo largo de las últimas tres semanas del mes de abril de 2019.

Análisis. Se realizó un análisis cuantitativo de los resultados con el programa SPSS 21.0. En primer lugar, se realizó un análisis descriptivo de las variables demográficas y las escalas relativas a Lenguaje Inclusivo. Además, se examinó la fiabilidad de las escalas a través del coeficiente alfa de Cronbach, como se indicó en el apartado anterior.

\section{RESULTADOS}

En primer lugar, se analizaron los estadísticos descriptivos y las relaciones entre las actitudes positivas hacia el Lenguaje Inclusivo, su uso y la edad (Tabla 1).

Tabla 1. Medias, desviaciones estándar y correlaciones de actitudes positivas, uso del Lenguaje Inclusivo y Edad.

\begin{tabular}{|c|c|c|c|c|c|}
\hline & M & DT & 1 & 2 & 3 \\
\hline 1. Actitudes positivas hacia el Lenguaje Inclusivo & 36.48 & 12.95 & - & $.812^{* *}$ & -.014 \\
\hline 2. Uso del Lenguaje Inclusivo & 7.60 & 3.10 & & - & -.052 \\
\hline 3. Edad & 32.75 & 12.45 & & & - \\
\hline
\end{tabular}

**. $p<.01 ; n=613$.

Nota. Los puntajes de las Actitudes Positivas hacia el Lenguaje Inclusivo oscilan entre 7 y 56 . Los puntajes del Uso del Lenguaje Inclusivo fueron de 3 a 15.

Como puede observarse en la Tabla 1, las actitudes hacia el Lenguaje Inclusivo se encuentran relacionadas con su uso $(r=.812 ; p<.01)$. Sin embargo, no se hallaron relaciones entre ninguna de estas variables y la edad de quienes participaron. 
Teniendo en cuenta los resultados de Parks y Roberton (1998, 2008), donde el grupo más joven había obtenido las puntuaciones más bajas en la variable de apoyo hacia el Lenguaje Inclusivo, se dividió la muestra en cuatro grupos etarios y se realizaron dos análisis de varianzas (ANOVA), a partir de los cuales se hallaron diferencias significativas. No obstante, el tamaño del efecto fue Bajo tanto para las actitudes positivas hacia el Lenguaje Inclusivo $\left(F_{(3,609)}=9.47, p<.01, \eta_{p}^{2}=.045\right)$ como para su uso $\left(F{ }_{(3,609)}=\right.$ $\left.11.85, p<.01, \eta_{p}^{2}=.055\right)^{6}$. El porcentaje de varianza explicada por el efecto de lá edad fue de $4.5 \%$ y $5.5 \%$ respectivamente.

Para conocer entre qué grupos se presentaban las diferencias, se realizó una prueba post-hoc de Tukey. Las cuatro agrupaciones se compusieron por los siguientes rangos de edad: 18 a 23 años (G1; $n=134)$, 24 a 34 años (G2; $n=284$ ), 35 a 49 años (G3; $n=100)$ y 50 a 70 años (G4; $n=95$ ). La prueba post-hoc indicó que las diferencias significativas en la evaluación de las actitudes agruparían al grupo más joven y al de mayor edad, por un lado, y, por otro, a los dos rangos etarios intermedios, los cuales tuvieron mayores actitudes positivas hacia el Lenguaje Inclusivo (Gráfico 1).

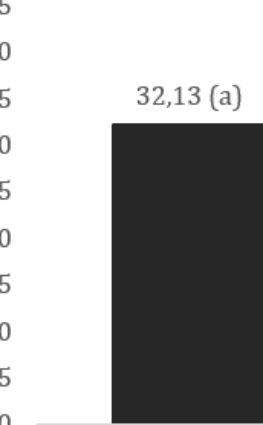

G1

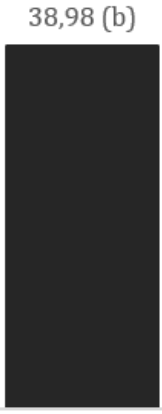

G2

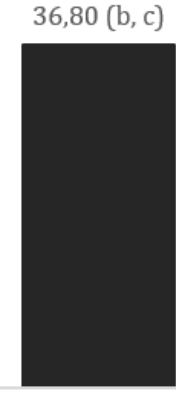

G3

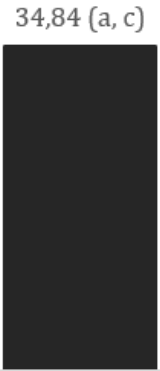

G4

Grupos etarios

Gráfico 1. Medias de Actitudes Positivas hacia el Lenguaje Inclusivo según Grupos Etarios. Nota. Las medias con distintas inscripciones difieren significativamente entre sí según la prueba post hoc de Tukey ( $p<.05)$.

En cuanto al uso, el grupo de 35 a 49 años no tuvo diferencias significativas con ninguno de los otros. Por otro lado, el grupo de 24 a 34 indicó un uso significativamente mayor que los grupos de menor y mayor edad (Gráfico 2 ).

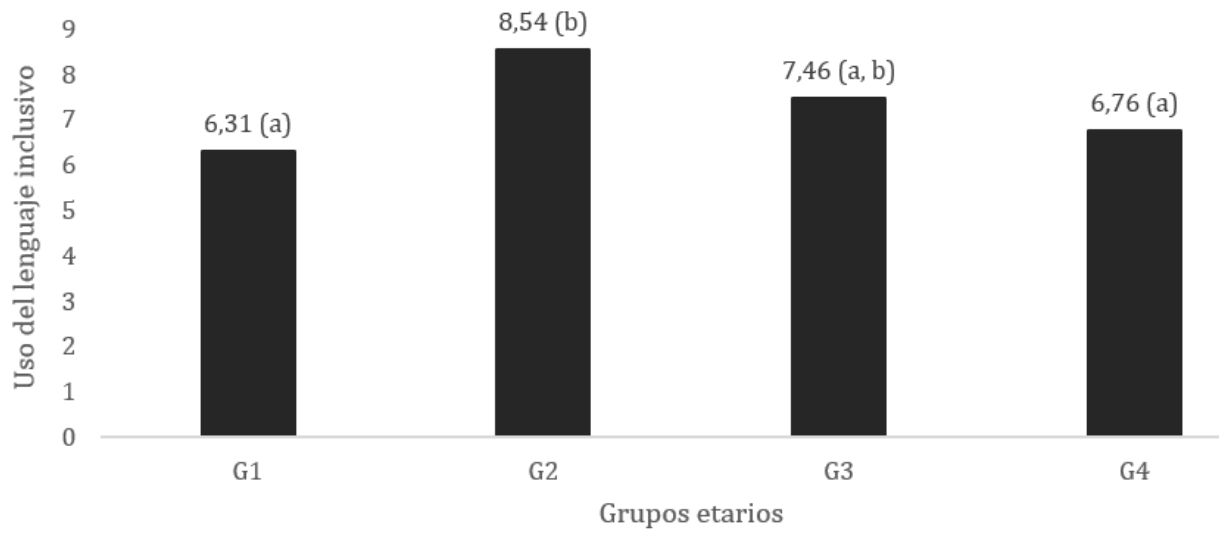

Gráfico 2. Medias de Uso del Lenguaje Inclusivo según Grupos Etarios.

Nota. Las medias con distintas inscripciones difieren significativamente entre sí según la prueba post hoc de Tukey ( $p<.05$ ). 
Posteriormente, se procedió a analizar si se hallaban diferencias entre la Identidad de Género, las actitudes hacia el Lenguaje Inclusivo y su uso (Tabla 2). Dentro de este análisis, no se incluyó al grupo de género no binario, debido al tamaño del grupo $(n=11)$.

Tabla 2. Actitudes Positivas hacia el Lenguaje Inclusivo y su uso según Género.

\begin{tabular}{ccccc}
\hline & \multicolumn{3}{c}{ Género } \\
\cline { 2 - 5 } & \multicolumn{2}{c}{ Femenino $(\mathbf{n}=\mathbf{3 4 6})$} & \multicolumn{1}{c}{ Masculino $(\mathbf{n}=\mathbf{2 5 6})$} \\
\cline { 2 - 5 } & $\mathbf{M}$ & $\mathbf{D T}$ & $\mathbf{M}$ & DT \\
\hline Actitudes positivas** & 38.99 & 12.05 & 33.27 & 13.17 \\
Uso del Lenguaje Inclusivo** & 8.24 & 3.91 & 6.66 & 3.88 \\
\hline
\end{tabular}

**. $\mathrm{p}<.01$.

Nota. Los puntajes de las actitudes positivas hacia el Lenguaje Inclusivo oscilan entre 7 y 56 ; Los puntajes del Uso del Lenguaje Inclusivo fueron de 3 a 15 .

Los dos géneros analizados tuvieron resultados con diferencias significativas para ambas escalas. El grupo que se identificó con el género femenino obtuvo una media más elevada en las escalas de actitudes positivas $(t=5.53 ; p<.01, d$ de Cohen $=.45)$ y uso $(t=$ $4.91 ; p<.01, d$ de Cohen $=.40$ ) del Lenguaje Inclusivo.

\section{DISCUSIÓN}

Este estudio tuvo como objetivos principales el análisis de la relación entre las actitudes hacia el Lenguaje Inclusivo y su uso con respecto al género y la edad de quienes participaron. En cuanto al género, los resultados indicaron que, en este contexto, aún existe cierta brecha en cómo evalúan y usan el Lenguaje Inclusivo los diferentes géneros. Las personas identificadas con el género femenino considerarían en mayor medida que este uso del lenguaje es relevante y que tiene consecuencias positivas para una mayor igualdad de los géneros, lo cual concuerda con la mayoría de las investigaciones sobre el tema (Douglas y Sutton, 2014; Gustafsson Sendén et al., 2015; Martyna, 1978; Parks y Roberton, 1998, 2008).

Esto podría deberse al mayor sentimiento de identificación y motivación que experimentan las personas del género femenino al interactuar con expresiones en Lenguaje Inclusivo. En su caso, el promedio de los resultados del grupo se encuentra cercano al acuerdo respecto al compromiso y la utilidad del uso del Lenguaje Inclusivo, mientras que, en el grupo de sujetos masculinos, la media supera muy levemente la afirmación "Ni de Acuerdo ni en Desacuerdo". Estos resultados implican una amplia potencialidad para la concientización sobre las consecuencias positivas del Lenguaje Inclusivo. A su vez, la elevada relación entre las actitudes y el uso de esta forma del lenguaje, indicaría que una intervención tendría consecuencias concretas en quienes sean foco de la actividad.

En cuanto al análisis de la relación de la edad con las variables evaluadas, en principio no se hallaron diferencias significativas. Sin embargo, sí se observaron diferencias al dividir la muestra en cuatro rangos etarios. Quienes tenían una edad de entre 18 y 23 años, pertenecientes a la cohorte $z$ o centennial, declararon tener menos actitudes positivas y un menor uso del Lenguaje Inclusivo. En este sentido no se diferenciaron del grupo etario de mayor edad (de entre 50 y 70 años), difiriendo con la hipótesis inicial de la investigación. Por otro lado, las actitudes y uso más elevado se hallaron en el grupo con edades de 24 a 34 años, donde se ubica la mayor parte de la generación y o millennial, el cual no tuvo diferencias con el grupo de 35 a 49 años. Los resultados de este trabajo 
coinciden en parte con los informados por Parks y Roberton (1998), quienes habían encontrado un mayor apoyo del Lenguaje Inclusivo en personas de mayor edad. Sin embargo, dividiendo la muestra en una mayor cantidad de grupos, observamos que esta tendencia se revierte en el grupo de más de 50 años. Por último, en lo que refiere al uso del Lenguaje Inclusivo, el grupo de 24 a 34 años declaró utilizarlo más frecuentemente que el grupo más joven y el de mayor edad.

Para poder fundamentar este comportamiento diferencial, en cuanto a la edad y las variables analizadas, sería necesario continuar indagando la relación del Lenguaje Inclusivo con un mayor abanico de variables. Estos resultados podrían provenir de una tendencia real de las generaciones intermedias a usar el Lenguaje Inclusivo, o bien podrían estar mediando otras variables como el nivel educativo y socioeconómico de quienes participaron. En Argentina, el Lenguaje Inclusivo fue acuñado y discutido en las universidades y círculos intelectuales; sin embargo, el actual movimiento feminista ha expandido su uso a un conjunto más amplio de la población.

Teniendo en cuenta las implicaciones del uso del masculino como genérico, el cual transmite sesgos y estereotipos que sobrerrepresentan al género masculino sobre los otros géneros, esta investigación buscó describir las relaciones del uso del Lenguaje Inclusivo con algunas variables demográficas.

Si bien los cambios en el lenguaje requieren de modificaciones complejas en toda la trama social, el desarrollo aquí presentado permitió realizar un aporte a la relación que tendría dicho proceso con la identidad de género y la particular relación con la edad. Además, es importante tener en cuenta aquellas investigaciones que indican que el tiempo desde la introducción de nuevos términos neutros o más igualitarios tiene un rol crucial en su aceptación, promoviendo su utilización a pesar de las resistencias iniciales. Se presentan como limitaciones a este trabajo el análisis únicamente de variables demográficas. Por otra parte, sería importante que próximos estudios hicieran hincapié en obtener respuestas de participantes con identidades de género no binarias, para analizar cuáles son las opiniones de esta población respecto a la temática.

\section{REFERENCIAS}

Aguirre, R. Ferrari, F. (2014). Las encuestas sobre uso del tiempo y trabajo no remunerado en América Latina y el Caribe. Caminos recorridos y desafíos hacia el futuro. Serie Asuntos de Género No122. CEPAL.

Bejarano Franco, M. T. (2013). El uso del lenguaje no sexista como herramienta para construir un mundo más igualitario. Vivat Academia, (124), 79-89. ISSN: 1575-2844

Budgeon, S. (2013). The Dynamics of Gender Hegemony: Femininities, Masculinities and Social Change. Sociology, 48(2), 317-334. https://doi. org/10.1177/0038038513490358

Campagnoli, M. A. (2014). iAndá a lavar los platos! Androcentrismo y sexismo en el lenguaje. En A. M. Bach (Ed.), Para una didáctica con perspectiva de Género, (pp. 59-106). Miño y Dávila - UNSAM.

Chenlo, N. (2014). Guía de Lenguaje Inclusivo en el Ministerio del Interior. Taller Gráfico. 
Douglas, K. M., Sutton, R. M. (2014). "A giant leap for mankind" but what about women? The role of system-justifying ideologies in predicting attitudes toward sexist language. Journal of Language and Social Psychology, 33(6), 667-680. https://doi. org/10.1177/0261927X14538638

Fishbein, M., Ajzen, I. (2011). Predicting and changing behavior: The reasoned action approach. Psychology Press.

Formanowicz, M. M., Cislak, A., Horvath, L. K., Sczesny, S. (2015). Capturing socially motivated linguistic change: how the use of gender-fair language affects support for social initiatives in Austria and Poland. Frontiers in psychology, 6, 1617. https://doi. org/10.3389/fpsyg.2015.01617

Frohard-Dourlent, H., Dobson, S., Clark, B. A., Doull, M., Saewyc, E. M. (2017). "I would have preferred more options": accounting for non-binary youth in health research. Nursing inquiry, 24(1), 24:e12150. https://doi.org/10.1111/nin.12150

Greenwald, A. G., Banaji, M. R. (1995). Implicit social cognition: attitudes, self-esteem, and stereotypes. Psychological review, 102(1), 4-27. https://doi.org/10.1037/0033295X.102.1.4

Gustafsson Sendén, M., Bäck, E. A., Lindqvist, A. (2015). Introducing a gender-neutral pronoun in a natural gender language: the influence of time on attitudes and behavior. Frontiers in Psychology, 6, 893. https://doi.org/10.3389/fpsyg.2015.00893

Hyde, J. S., Bigler, R. S., Joel, D., Tate, C. C., van Anders, S. M. (2019). The future of sex and gender in psychology: Five challenges to the gender binary. American Psychologist, 74(2), 171. https://doi.org/10.1037/amp0000307

Jiménez Rodrigo, M. L., Román Onsalo, M. L., Traverso Cortés, J. (2010). Diagnóstico sobre la sensibilidad hacia el lenguaje no sexista por parte del alumnado universitario. En Investigaciones multidisciplinares en Género: II Congreso Universitario Nacional Investigación y Género. Facultad de Ciencias del Trabajo de la Universidad de Sevilla, 17 y 18 de junio de 2010. (Coord.) Isabel Vázquez Bermúdez; (Com. cient.) Consuelo Flecha García et al. (567-585). Sevilla: Unidad para la Igualdad, Universidad de Sevilla.

Jiménez Rodrigo, M. L., Román Onsalo, M. L., Traverso Cortés, J. (2011). Lenguaje no sexista y barreras a su utilización. Un estudio en el ámbito universitario. Revista de investigación en educación, 2(9), 174-183.

Kaufmann, C., Bohner, G. (2014). Masculine generics and gender-aware alternatives in Spanish. IFFOnZeit-Online Journal of the Interdisciplinary Center for Research on Women and Gender at the University of Bielefeld, 4(3), 8-17.

Knussen, C., Yule, F., MacKenzie, J., Wells, M. (2004). An analysis of intentions to recycle household waste: The roles of past behaviour, perceived habit, and perceived lack of facilities. Journal of Environmental Psychology, 24(2), 237-246. https://doi. org/10.1016/j.jenvp.2003.12.001

Lindqvist, A., Renström, E. A., Sendén, M. G. (2019). Reducing a male bias in language? Establishing the efficiency of three different gender-fair language strategies. Sex Roles, 81(1-2), 109-117. https://doi.org/10.1007/s11199-018-0974-9 
Maass, A., Arcuri, L. (1996). Language and stereotyping. In N. Macrae, M. Hewstone, \& C. Stangor (Eds.), Stereotypes and stereotyping (pp. 193-226). Guilford Press.

Martyna, W. (1978). What does 'he' mean? Use of the generic masculine. Journal of communication, 28(1), 131-138. https://doi.org/10.1111/j.1460-2466.1978. tb01576.x

Parks, J. B., Roberton, M. A. (1998). Influence of age, gender, and context on attitudes toward sexist/nonsexist language: Is sport a special case? Sex roles, 38(5-6), 477494. https://doi.org/10.1023/A:1018766023667

Parks, J. B., Roberton, M. A. (2008). Generation gaps in attitudes toward sexist/nonsexist language. Journal of Language and Social Psychology, 27(3), 276-283. https://doi. org/10.1177/0261927X08317956

Puppo, M. L. (2009). Repensar el mundo a partir de los textos. En S. Elizalde, K. Felitti, y G. Queirolo (Eds.). Géneros y sexualidades en las tramas del saber. Revisiones y Propuestas. Libros del Zorzal.

Sarrasin, O., Gabriel, U., Gygax, P. (2012). Sexism and attitudes toward gender-neutral language. Swiss Journal of Psychology, 71, 113-124. doi: 10.1024/1421-0185/ a000078

Sato, S., Athanasopoulos, P. (2018). Grammatical gender affects gender perception: Evidence for the structural-feedback hypothesis. Cognition, 176, 220-231. https:// doi.org/10.1016/j.cognition.2018.03.014

Sczesny, S., Formanowicz, M., Moser, F. (2016). Can gender-fair language reduce gender stereotyping and discrimination? Frontiers in Psychology, 7, 25. https://doi. org/10.3389/fpsyg.2016.00025

Sczesny, S., Moser, F., Wood, W. (2015). Beyond sexist beliefs: How do people decide to use gender-inclusive language? Personality and Social Psychology Bulletin, 41(7), 943-954. https://doi.org/10.1177/0146167215585727

Stahlberg, D., und Sczesny, S. (2001). Effekte des generischen Maskulinums und alternativer Sprachformen auf den gedanklichen Einbezug von Frauen. [Efectos del masculino genérico y formas alternativas del lenguaje en la inclusión mental de la representación de las mujeres]. Psychologische Rundschau, 52(3), 131-140.

Stout, J. G., \& Dasgupta, N. (2011). When he doesn't mean you: Gender-exclusive language as ostracism. Personality and Social Psychology Bulletin, 37(6), 757-769. https://doi.org/10.1177/0146167211406434

Vervecken, D., Hannover, B., Wolter, I. (2013). Changing (S) expectations: How gender fair job descriptions impact children's perceptions and interest regarding traditionally male occupations. Journal of Vocational Behavior, 82(3), 208-220. https://doi. org/10.1016/j.jvb.2013.01.008 